\title{
T2-mapping and T1-mapping detect myocardial involvement inTako-Tsubo cardiomyopathy: a preliminary experience
}

\author{
Emmanuelle Vermes*, Lauriane Pericart, Julien Pucheux, Anne Delhommais, Daniel Alison, Olivier Genee \\ From 18th Annual SCMR Scientific Sessions \\ Nice, France. 4-7 February 2015
}

\section{Background}

T2- and T1-mapping are novel CMR techniques allowing objective tissue characterization. These techniques have been shown to be superior to dark blood imaging in NSTEMI patients in detecting ischaemic area at risk and acute oedema. These methods have not been assessed in Tako-Tsubo cardiomyopathy (TC).The aim of the study was to assess myocardial involvement using T2- and T1mapping in Tako-Tsubo cardiomyopathy (TC).

\section{Methods}

Nine patients with TC and 15 controls were prospectively enrolled. Cardiovascular magnetic resonance (CMR) was performed a mean 2.8 days after the onset of symptoms and after a mean 4.6 month follow-up. CMR was applied using T2-mapping, pre and post contrast T1-mapping (MOLLI) and LGE sequences. Segmental and global $\mathrm{T} 1$ values have been measured before and after contrast administration. Wall motion abnormality (WMA) was assessed.

\section{Results}

All patients were female, had positive troponin $(6 \pm 9 \mu \mathrm{g} / \mathrm{l})$ and medium and/or apical ballooning associated with moderate LV dysfunction (EF $44 \pm 7 \%$ ). On admission, compared with controls, TC patients had significantly higher T2 values $(65 \pm 6 \mathrm{~ms}$ vs $50 \pm 4 \mathrm{~ms}, \mathrm{p}<0.0001)$. Myocardial T2 was significantly higher in segments with WMA compared to normokinetic segments $(67 \pm 12 \mathrm{~ms}$ vs $61.5 \pm 8 \mathrm{~ms}, \mathrm{p}=0.003)$. Compared with controls, TC patients had significantly higher pre contrast $\mathrm{T} 1$ values $(1115 \pm 92$ versus $1016 \pm 89, \mathrm{p}<0.0001)$ and significantly lower post contrast T1 values $(428 \pm 24 \mathrm{~ms}$ vs $466 \pm 19$ ms, $\mathrm{p}=0.02$ ).Pre contrast $\mathrm{T} 1$ values were significantly higher in segments with WMA compared to normal segments $(1126 \pm 95$ vs $1089 \pm 85, \mathrm{p}=0.016)$. Post contrast $\mathrm{T} 1$ values were not significantly different in abnormal segments compared to normal segments ( $421 \pm 56$ vs 431 $\pm 50, p=0.15$ ). No patients had LGE (assessed visually). At follow-up: all had a complete LV recovery (EF: 67 $\pm 4 \%)$ without significant WMA. Mean T2 and pre contrast $\mathrm{T} 1$ values decreased significantly at follow up (53 $\pm 6 \mathrm{~ms}$ vs $65 \pm 8 \mathrm{~ms}, \mathrm{p}=0.001$ and $1016 \pm 76$ vs $1115 \pm 80$, $\mathrm{p}=0.001$ respectively). No differences were observed regarding post contrast global $\mathrm{T} 1$ values.

\section{Conclusions}

In TC patients, T2-mapping and pre contrast T1-mapping allow identification of reversible myocardial injury. Post contrast T1 mapping does not provide additional information.

\section{Funding}

No funding.

Published: 3 February 2015

doi:10.1186/1532-429X-17-S1-P354

Cite this article as: Vermes et al:: T2-mapping and T1-mapping detect myocardial involvement inTako-Tsubo cardiomyopathy: a preliminary experience. Journal of Cardiovascular Magnetic Resonance 2015 17(Suppl 1):P354. 